Literatura y Lingüística $\mathrm{N}^{\circ} 24$

ISSN 0716-5811 / pp. 81-96

\title{
Un viaje por el infierno, de Alberto Gamboa: escritura testimonial e imaginario de la reconciliación*
}

\author{
Jaume Peris Blanes**
}

\section{Resumen}

El artículo analiza el testimonio Un viaje por el infierno, de Alberto Gamboa, que articuló el imaginario periodístico del que provenía su autor y la escritura testimonial que había sido de gran importancia en el exilio, pero que había carecido de espacios de expresión en el Chile de la dictadura. El autor analiza el modo en que el testimonio de Gamboa puso en contacto por primera vez las características enunciativas de las escrituras testimoniales con el imaginario de la reconciliación nacional.

Palabras clave: Testimonio, dictadura chilena, reconciliación nacional, libroreportaje

\section{Un viaje por el infierno by Alberto Gamboa: testimonial writing and imaginary of the Reconciliation}

\begin{abstract}
The author focuses on the testimony Un viaje por el infierno, written by the survivor Alberto Gamboa, which articulated the imaginary of journalism based on his testimonial writing. Moreover, it relates, for the first time, to the main features of testimonies to the imaginary of national reconciliation.
\end{abstract}

Key words: Testimony, Chilean dictatorship, national reconciliation, journalism

Recibido: 24-03-2011 Aceptado: 11-04-2011

El trabajo se enmarca en una investigación postdoctoral más amplia en torno a los discursos sobre la represión y los imaginarios de la memoria en el Cono Sur latinoamericano.

** Profesor de literatura latinoamericana de la Universitat de València (España) departamento de Filología Española. Doctor en Filología. Dirección electrónica: Jaume.peris@uv.es 
Un viaje por el infierno, de Alberto Gamboa: escritura testimonial e imaginario de la reconciliación / Jaume Peris Blanes

\section{Introducción}

La publicación de Un viaje por el infierno (1984), testimonio del periodista Alberto 'Gato' Gamboa, constituyó un hecho insólito en la escena cultural de los primeros años ochenta. En primer lugar, porque se trataba de un superviviente de un campo de concentración que exponía públicamente en Chile su relato de lo vivido en el periodo de tiempo de su encierro, de un modo similar a como lo habían hecho, unos años antes, los supervivientes en el exilio. En segundo lugar, porque Gamboa anudaba la enunciación testimonial a una publicación periódica y, en cierta medida, a algunas formas y rasgos asociados a la tradición minoritaria del periodismo chileno que, en los años anteriores, se había identificado con los emergentes discursos de defensa de los derechos humanos.

El objetivo de este artículo es analizar el modo en que la forma testimonial se articuló, en ese contexto, a las pautas discursivas que se habían ido gestando en los discursos de oposición al régimen, y en especial en los reportajes periodísticos que habían hecho públicas las desapariciones y las luchas de los familiares por hallar a los detenidos desaparecidos. En particular, el objetivo es describir históricamente la forma específica en que se articularon los rasgos principales de la enunciación testimonial (descritos, entre otros, por Agamben, 2000) al imaginario de la reconciliación nacional que, en esos años, estaba sosteniendo las intervenciones de oposición al régimen militar en materia de derechos humanos (Cruz, 2004; García Castro, 2002, Moors, 1994).

Para ello, el autor se valdrá del análisis textual del testimonio de Gamboa, sirviéndose de las herramientas metodológicas desarrolladas en trabajos anteriores (Peris Blanes 2005 y 2008) e inscribiendo dicho texto en un contexto de intervenciones más amplio, que indique su relación con las condiciones ideológicas y discursivas del momento, de acuerdo a la perspectiva histórica y social del testimonio trazada por Wieviorka:

El testimonio, sobre todo cuando se halla integrado en un movimiento de masas, expresa, además de la experiencia individual, el o los discursos que tiene la sociedad, en el momento en que el testigo cuenta su historia, sobre los acontecimientos que el testigo ha vivido. Dice, en principio, lo que cada individuo, cada vida, cada experiencia de la Shoa tiene de irreductiblemente único. Pero lo dice con las palabras que son propias de la época en que testimonia, a partir de un cuestionamiento y de unas expectativas que son también contemporáneas de su testimonio, asignándole finalidades dependientes de intereses políticos o ideológicos, 
contribuyendo así a crear una o más memorias colectivas, erráticas en su contenido, en su forma, en su función y en la finalidad, explícita o no, que ellas se asignan $(1998,13)$.

\section{Periodismo y testimonio: la retórica de la reconciliación}

El testimonio de Gamboa, a pesar de su singularidad textual, se inscribía en una serie de publicaciones que, en los primeros años de la década, habían sacado a la luz, en condiciones de represión, censura y precariedad editorial, el funcionamiento del sistema represivo y, en especial, las políticas de desaparición forzada de los primeros años del régimen militar. Se trataba de publicaciones ligadas al ámbito del periodismo que, bajo el signo del libro-reportaje, se nutrían de los documentos, declaraciones y testimonios contenidos en el archivo de la Vicaría de la Solidaridad, reelaborándolos para construir reportajes en torno a casos específicos de desapariciones. Los primeros fueron Lonquén, de Máximo Pacheco, y Detenidos-Desaparecidos, una herida abierta, de Patricia Verdugo y Claudio Orrego, cuya publicación fue demorada por la censura desde 1980 a 1983, pero en los años siguientes verían la luz publicaciones como Nunca Más Chile. 1973-1984 [1984, edición censurada] (1986), de Myriam Pinto o Miedo en Chile (1985) de Patricia Politzer.

En ellos se daban cita historias de desapariciones, de la búsqueda de los desaparecidos por parte de sus familiares y relatos de la experiencia vivida en los campos de concentración por parte de algunos de sus supervivientes. Su estructura enunciativa, sin embargo, basculaba entre la necesidad de incluir la voz de las víctimas tal como habían aparecido en las entrevistas o en los documentos del archivo y la voluntad de construir una voz de denuncia en la que la heterogeneidad de las voces cobraran sentido y organicidad narrativa y, de ese modo, potenciaran su efecto de denuncia en el escenario del Chile dictatorial (Unnold, 2002).

Estos libros habían sido precedidos por las intervenciones de la propia Vicaría a través de sus publicaciones periódicas y por los diferentes volúmenes de ¿Dónde están?, que habían presentado públicamente antecedentes relacionados con múltiples casos de desapariciones. Esas publicaciones, en un contexto de máxima dificultad, debieron envolverse en una difícil estrategia retórica para poder ver la luz, haciendo que sus demoledoras condenas del sistema represivo fueran siempre acompañadas de un reconocimiento -simulado o real, poco importa- 
Un viaje por el infierno, de Alberto Gamboa: escritura testimonial e imaginario de la reconciliación / Jaume Peris Blanes

de la labor del gobierno y de una supuesta ingenuidad en torno a los fines políticos de la violencia ${ }^{1}$. La idea de reconciliación nacional, que había formado parte del discurso de la Iglesia desde el golpe militar, sirvió para abrochar discursivamente ese delicado engranaje retórico y para legitimar una estrategia que el régimen militar no había dudado en tachar de politizada y, en un primer momento, de cómplice con la clandestinidad.

La articulación entre esa tonalidad conciliatoria y la durísima crítica subyacente a las publicaciones de la Vicaría condensaba, en realidad, la estrategia que la Iglesia Católica llevó a cabo en la primera etapa del gobierno militar, y que la especialista María Angélica Cruz cifra en la idea de un 'doble juego' tanto por parte de la Iglesia como del gobierno militar:

por un lado, la jerarquía eclesial aceptó las reglas del nuevo gobierno de facto, ofreciendo además su apoyo para favorecer la 'reconciliación' entre los chilenos; por el otro, desarrolló una labor de asistencia a las víctimas de la represión que ese mismo gobierno perseguía. A su vez, desde el Estado se buscó el respaldo de la Iglesia, mientras paralelamente se reprimía a quienes dentro de ella eran vistos como 'enemigos' del régimen (2004: 3).

Lo cierto es que a partir de 1980 la postura de la Iglesia cambiaría y articularía públicamente una abierta denuncia de las políticas sociales, económicas y represivas del régimen militar. La publicación en 1978 del primer tomo de ¿Dónde están? se enmarcaba por tanto en un periodo de transición en la postura de la Iglesia, pero la retórica conciliatoria que, a pesar de todo, había estructurado sus denuncias a la Junta se convertiría en un elemento fundamental de las políticas de denuncia que, en el interior de Chile, iban a tratar de hacer visible el carácter de la represión militar.

Los libros-reportaje a los que hacíamos referencia antes reproducían con distintos matices la retórica de la reconciliación ensayada por la Vicaría, en un momento en que el gobierno militar ponía todos sus

1 Tal como analiza Naomi Klein (2007), la estrategia de no señalar el carácter político de la violencia y desvincular la defensa de los derechos humanos de la crítica al sistema socio-económico al que era funcional el sistema represivo, permitió al capitalismo neoliberal liberarse de su vinculación a la violencia extrema desplegada en las dictaduras militares de los setenta. En el caso de Chile, esa desvinculación, que se hallaba presente en los movimientos de defensa de los derechos humanos y en las intervenciones de la Vicaría, sirvió para que las denuncias de la violencia pudieran ver la luz en su momento, pero más tarde, con la llegada de la democracia, determinaron el sesgo de las políticas de memoria y la consolidación pública del modelo neoliberal, que se eximía así de su responsabilidad en la violencia represiva chilena. 
esfuerzos en la institucionalización del régimen e, incluso, se apuntaba en ocasiones a la idea de la reconciliación nacional. La novedad de los libros-reportaje con respecto a las sucesivas entregas del ¿Dónde están? (1978) radicaba en que los documentos no aparecían en bruto, sino transmutados a otra matriz narrativa que podía acercarlos a otro tipo de público (Moors, 1994)2. También, en que esos documentos salían definitivamente del ámbito de la Vicaría y eran incorporados al ámbito profesional del periodismo.

En ese contexto de emergencia de discursos públicos de denuncia, el texto de Gamboa fue publicado en cuatro tomos que acompañaron a cuatro ediciones sucesivas de la revista Hoy, que había apostado por abrir una colección de testimonios que complementara con las voces de las víctimas de la violencia el análisis crítico que tenía lugar en sus páginas ${ }^{3}$. Ello suponía la inclusión definitiva de la escritura testimonial en la escena periodística, haciendo público el testimonio de un superviviente que, además, aparecía estrechamente vinculado a una concepción del periodismo como un elemento de la lucha social. Gamboa, antiguo director de Clarín en los tiempos de Allende, confirmaba constantemente esa vinculación entre la escritura testimonial y la labor periodística:

No me ha costado escribir lo que he visto porque he trabajado como si fuera reportero. No me puse en un carácter de ensayista, pues me da la impresión de que quien se pone en este plano le cuesta más (1984, I: 6).

Pero esa posición periodística, en tanto que se aliaba a la condición de superviviente, adquiría un cariz singular:

¿Es justo cerrar así este capítulo de mi vida y de la vida de tantos otros compatriotas, con un silencio total? Honestamente, pienso que no. ¿Qué hacer, entonces? No hay que ser brujo para encontrar la respuesta. Contar lo que vi. Es decir, lo que hice como reportero. Vivir la noticia. Y después de vivirla, escribirla apegada fielmente a los hechos. (...) Todo lo que pretendo contar tiene un montaje humano. ¡Absolutamente humano! (...) Este amplio

2 Ximena A. Moors (1994) señaló el carácter ambulatorio de los documentos contenidos en el archivo de la Vicaría de la Solidaridad, incluidos los testimonios, que fueron desplazados a diversas matrices discursivas como el libro-reportaje, el teatro, el cine o la entrevista para hacer llegar al público una documentación de un gran valor histórico.

3 En la Introducción, Mauricio Carvallo señalaba la voluntad de continuidad de esta colección: "Alberto 'Gato' Gamboa Soto aprovecha el lanzamiento de la serie 'Testimonios' de HOY, por cuya puerta es el primero en entrar. Y la dejará abierta a otros chilenos que nos contarán a nosotros y a la historia, lo que ignorábamos -o queríamos ignorar- cuando hacíamos una existencia normal, mientras a ellos les cambiaban sus vidas" (1984, I: 7). 
Un viaje por el infierno, de Alberto Gamboa: escritura testimonial e imaginario de la reconciliación / Jaume Peris Blanes

reportaje, novelado a veces y otras veces no, no tiene ambiciones literarias. Es simplemente un testimonio. ¡Que se entienda bien! Un testimonio y no una acusación. No he sido ni soy el dueño de la verdad, como se estila en estos momentos (I: 14).

Gamboa resituaba así una problemática a la que habían debido enfrentarse los libros-reportaje que le habían precedido: el de la difícil relación entre la voz del testigo y la figura legitimadora del periodista de investigación. Haciéndolas coincidir, literalizaba la identificación-que en los textos anteriores había tenido un cariz más metafórico- entre el periodista en búsqueda de la verdad y el saber de los supervivientes que éste autorizaba en sus investigaciones.

El testimonio de Gamboa, por tanto, integraba de forma sorprendente los dos paradigmas principales sobre los que se estaban articulando las denuncias públicas más importantes tanto en el interior de Chile como en el exilio, donde los supervivientes habían adquirido una gran centralidad, a lo largo de los años setenta, en las estrategias de denuncia al régimen militar ${ }^{4}$. Un viaje por el infierno era, pues, un texto que compartía gran parte de las características enunciativas de los testimonios del exilio pero que las desplazaba hacia una economía de representación propia de una cierta tradición periodística.

El testimonio fue dividido en cuatro entregas de una extensión idéntica (96 páginas) que se distribuyeron de forma periódica como suplementos de la revista, pautando sus tiempos de lectura y de distribución de información -al modo de las novelas o reportajes por entregas- y envolviendo la voz testimonial en una iconografía determinada-vinculada a una cierta tradición del cómic o de la estampa periodística- que inscribía en un tono específico el relato de los avatares de Gamboa en los campos de concentración.

De hecho, los dibujos que puntuaban el texto trataban de fijar en imágenes bastante codificadas por la tradición del cómic algunas de las reflexiones de Gamboa y algunas de las situaciones que describía en su relato. El tono sobrio pero profundamente melancólico de sus dibujos suponía un importante suplemento a la significación del texto, así como sus llamativas portadas y el formato de media cuartilla en el que se editó. Todo ello permitía inscribir la voz testimonial en una matriz de intervención pública que aparentemente era ajena a su posición enunciativa pero que en los últimos años se estaba postulando como la única capaz

4 La función desempeñada por los testimonios de supervivientes en el exilio ha sido analizada con detalle en Peris Blanes (2008). 
de inscribir, aunque de un modo desplazado, la voz de los supervivientes en el espacio público.

\section{Experiencia traumática y fragmentación del relato}

El texto planteaba cuestiones novedosas con respecto a los anteriores testimonios publicados en Chile. Por una parte, su extensión le permitía desplegar un proyecto narrativo global en el que la experiencia extrema vivida en los campos fuera elaborada en una narración, aunque ésta presentara una debilidad estructural similar a la de la mayoría de los testimonios del exilio (Dorfman, 1986). El texto se articulaba en torno a escenas fragmentadas de las que se extraía una cierta enseñanza, pero que no estaban articuladas en torno a un principio de causalidad o continuidad narrativa. Sin embargo, el superviviente aprovechaba muy conscientemente la segmentación editorial del texto que le imponía el tipo de publicación por entregas para inscribir las partes de su testimonio en cuatro de las funciones fundamentales del relato tradicional, siendo la primera y la última "La partida" y "El regreso" y reservando para las dos partes centrales la vida cotidiana en los campos (en el "Infierno") con "Chacabuco" y "Vida de perros".

Se generaba, así, una cierta tensión entre la inscripción del testimonio en los moldes del relato clásico -en el que, necesariamente, un aprendizaje global, expresado narrativamente en las transformaciones subjetivas, debería tener lugar- y su articulación en torno a escenas fragmentarias y personajes sin continuidad narrativa. De hecho, si bien el texto trataba de ordenar la experiencia concentracionaria siguiendo una ordenación cronológica, lo cierto es que en muchos momentos -cuando se centraba en aspectos de la cotidianidad del campo o en historias y perfiles de otros detenidos- ese modelo de organización se disgregaba, rompiéndose recurrentemente por la emergencia de ordenamientos horizontales, con integraciones narrativas autónomas al relato global.

Ese tipo de construcción textual hacía visible la dificultad del superviviente para incluir su experiencia en una continuidad causal, a pesar de la estructura de relato tradicional en la que parecían incluirla los títulos del primer y último tomo ('La partida' y 'El regreso'). Es ese un tipo de estructura típica de la escritura testimonial, que no sin dificultad trata de dar un sentido temporal y vivencial a una experiencia traumática que aparece, en lo esencial, separada del resto de sus vidas. Planteó Lawrence Langer, en un libro ya clásico, que: 
Un viaje por el infierno, de Alberto Gamboa: escritura testimonial e imaginario de la reconciliación / Jaume Peris Blanes

el testimonio es una forma de memoria (...) luchando contra lo que podemos llamar memoria disruptiva, es un esfuerzo de reconstruir algo similar a una continuidad en la vida como si fuera una existencia normal. La 'cotemporalidad' deviene el principio de esos testimonios, en tanto que el testigo lucha con la imposible tarea de hacer que sus reminiscencias del campo se articulen con el resto de sus vidas (Langer 1991: 3).

Por ello, la débil narratividad de la mayoría de estos testimonios no debe leerse como un fracaso estético, sino como una señal del modo en que los supervivientes trataron de articular en el discurso la experiencia vivida en los campos. De hecho, la mayoría de estos testimonios careció de una línea de continuidad causal que hiciera inteligibles las relaciones entre unos y otros elementos narrativos. En definitiva, lo que faltó en gran parte de estos textos fue un sujeto fuerte capaz de suturar con su presencia la disgregación de los diversos elementos de la experiencia que estaban poniendo en juego en sus relatos.

Ello nos habla, a mi entender, de dos cuestiones fundamentales: en primer lugar el hecho de que la experiencia vivida en los campos se presentaba de modo totalmente fragmentado a los supervivientes; y en segundo lugar, que al elaborar narrativamente esa experiencia fragmentada los supervivientes no hallaron la forma, en la mayoría de los casos, de integrar esos elementos disgregados en una narración unitaria ${ }^{5}$.

\section{Dignificación de los detenidos y representación de la violencia}

El testimonio de Gamboa se abría con una demanda de reconocimiento de su carácter de víctima arbitraria de las políticas represivas de la junta, y de la ausencia de razones jurídicas y lógicas para ser sometido al castigo de la vida concentracionaria. Tras presentar en facsímil el Certificado de las fuerzas Armadas en que constaba que había sido liberado sin ningún cargo, abría el relato con las siguientes palabras:

A partir de 1975 [de su liberación], este documento es mi carta de presentación. Asegura que no alteré las normas constitucionales, no rompí con el orden establecido y fui un riguroso observante

5 Desde un punto de vista teórico, las contradicciones de la enunciación testimonial están analizadas en Peris Blanes (2005) siguiendo las ideas de Agamben (2000).

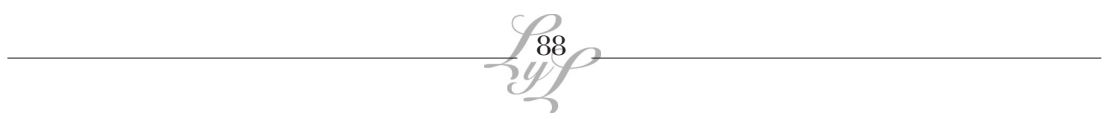


del decreto exento. Así lo sostiene este certificado oficial. En resumen, que soy una blanca paloma (1984, I: 13).

No sin ironía se servía Gamboa del modo en que las fuerzas armadas 'legalizaban' su situación tras la liberación para socavar los discursos de legitimación que justificaban su actuar, negando la ecuación entre 'detenido' y 'peligro público' en que estos se basaban. Ese gesto se refrendaba durante todo el relato, que insistía en una representación de los presos políticos 'como seres humanos, con sus grandezas y sus debilidades' (1984, I: 14), tratando de dignificar la imagen de los presos y de combatir el estigma social que sobre ellos cayera tras su liberación. Trataba, pues, de habilitar la condición de 'víctima de la violencia de Estado', si no como estatuto jurídico, sí, al menos, como elemento de reconocimiento social.

Esa fue una de las funciones públicas que estas intervenciones periodísticas desempeñaron, y ello estuvo siempre acompañado de una continua reflexión sobre el hecho mismo de testimoniar. Como si teorizar el testimonio sirviera, además, para dignificar a sus autores potenciales. En primer lugar, planteando las dificultades de la enunciación testimonial para hacer frente a la experiencia traumática de los campos: "¿Cómo describir el miedo? ¡Puchas la cosa difícil! Sobre todo en esas circunstancias" (1984, I: 35). Y ligando esa dificultad a los efectos que la violencia producía en la subjetividad del preso:

¿Pero, qué hace el miedo pasando tan velozmente del motor de las ideas al motor de los sentimientos? ¿Y qué tienen que ver mis piernas en todo esto? Varias veces reflexioné largamente sobre este asunto. Cada vez que fui aislado en un lugar solitario tuve tiempo para pensar lo que quisiera sobre el tema. Más adelante, cuando pasé por otras experiencias más duras, tuve que agregar forzosamente a este puzzle otras zonas de mi cuerpo. Por ejemplo, el estómago. Cuando atisbas el peligro se te hace como un nudo en el estómago. Pero no un nudo ciego hecho con una pitilla, sino que un nudo ciego con una soga o una cadena, que te hace un bulto gigantesco, que pesa una brutalidad y se mueve para todos los lados. La boca también juega un papel importante. Se te seca de un viaje cuando estás en apuros. Y no hay manera de fabricar saliva. (...) Tampoco puedo dejar fuera de estas reflexiones a la nuca. Con la nuca la gente hace muchas bromas que tienen que ver con las infidelidades. Pero la nuca para un hombre detenido es como el radar de todas sus reacciones. (1984, I: 36) 
Un viaje por el infierno, de Alberto Gamboa: escritura testimonial e imaginario de la reconciliación / Jaume Peris Blanes

Es sabido que la lógica concentracionaria busca producir en los detenidos una angustiosa sensación de fragmentación corporal a través de la aplicación del dolor extremo o del miedo ${ }^{6}$. En el fragmento precedente, esta angustia de fragmentación se representaba de un modo bastante sorprendente: por una parte, Gamboa describía los procesos físicos que le producía el miedo dando a los diferentes elementos corporales una figuración atomizada, haciendo que aparecieran como elementos autónomos desconectados de un esquema global; por otra parte, la voz testimonial abordaba ese proceso con una fuerte voluntad de impersonalización, adoptando una posición analítica que otorgaba un carácter general a sus observaciones.

De hecho, pareciera que el sujeto de ese proceso no fuera el superviviente Alberto Gamboa, sino el 'hombre detenido' en tanto que ente abstracto. Ese desplazamiento de la angustia hacia una abstracción mucho mayor tenía que ver, por una parte, con la voluntad programática de este testimonio de recuperar la experiencia colectiva de la concentración y, por tanto, con hacer de las vivencias subjetivas una metonimia perfecta de las vivencias de la comunidad de presos. Pero era también una forma de construir una importante distancia entre el sujeto de la enunciación y los procesos desgarradores de los que estaba tratando de dar cuenta. Una distancia que permitiera objetivar en lo posible una experiencia que amenazaba con destruir a la subjetividad misma (Agamben, 2000).

Ese juego de distancias apuntaba directamente al estatuto que la escritura testimonial tenía en el texto, y a los diferentes tiempos de elaboración de la experiencia traumática. De hecho, Gamboa contaba brevemente cómo su texto tenía como origen las notas tomadas en el interior del campo de Chacabuco, que servirían más tarde (ya liberado) de material de base para la construcción del testimonio. Ello parece explicar algo de la estructura narrativa antes señalada: es más que probable que la segmentación del texto en escenas casi autónomas y en perfiles individuales tuviera como origen la fragmentación de las notas tomadas in situ por el superviviente, y la dificultad para homogeneizarlas sin traicionar el tono de éstas y el tipo de experiencia que lo determinaba.

Pero también apuntaba a la propia relación que el superviviente mantenía, en el momento de la escritura final (y a la hora de construir su voz pública como testigo) ante la memoria de la experiencia concentracionaria y hacia los diferentes elementos que la disparaban: "Recordé

$6 \quad$ Puede consultarse una interesante reflexión sobre el cuerpo concentracionario en Sucasas (2000) o el trabajo ya clásico de Elaine Scarry sobre la lógica de la tortura (1985). 
este episodio, medio trágico, medio cómico, porque se había quedado olvidado en mis apuntes" (1984, II: 47). Gamboa no se extendería en los avatares de ese proceso ("este libro que durante largos diez años ordené y reordené en mi mente y en mi corazón” [1984, IV: 94]), pero en el texto quedaban, sin embargo, huellas de esa doble temporalidad de la escritura (Derrida 2005) que, aunque de forma no especialmente remarcada, atravesaba subterráneamente todo el testimonio.

\section{Metáfora familiar e ideología de la reconciliación}

El testimonio de Gamboa se presentaba, además, como un puente intergeneracional: el libro estaba dedicado a su hijo Alberto, a quien ubicaba en el paradigma del 'compañero'. Pero a la vez, en la misma hoja de la dedicatoria, señalaba la existencia de alguien que, en los días de encierro, había sufrido más que él mismo: se trataba de su 'anciano padre', a quien también dedicaba el libro. Así, el texto elegía como interlocutores simbólicos a los integrantes del núcleo familiar entre los cuales generacionalmente se situaba, haciendo hincapié en su lugar como puente entre ellos y en el rol que, como experiencia fundamental, había tenido la estancia en los campos de concentración en el interior de su familia. El tono esperanzado de las dedicatorias se complementaba con la introducción realizada por Mauricio Carvallo, en la que se leía que se glorificaba su 'optimismo' como actitud cívica ejemplar y la idea de 'reconciliación' como principio básico de las políticas futuras.

El carácter de esos paratextos conectaba directamente con una de las escenas más importantes del libro, en la que se detallaba un encuentro que iba a determinar la voluntad del superviviente de escribir y hacer público su testimonio de la vida en el campo. Señalaba Gamboa la llegada al campo de Chacabuco de un capellán de carabineros encargado, entre otras tareas, de leer las cartas de los detenidos para encontrar motivos posibles de censura. Y relataba el encuentro que ambos mantuvieron al día siguiente de enviarle a su mujer una hermosa carta en que cruzaba el análisis de la cotidianeidad del campo con una constante demanda de amor:

Caminamos como dos cuadras, conversando amistosamente. Era un buen cura. Le gustaban la música, la poesía, la lectura. Tal vez por eso, era mucho más amigo del sector intelectualizado de Chacabuco. No soportaba el trato inhumano ni los vejámenes que se diera a los presos. Protestaba cuando debía protestar. La mayoría de las veces, estérilmente. La máquina del odio tenía 
Un viaje por el infierno, de Alberto Gamboa: escritura testimonial e imaginario de la reconciliación / Jaume Peris Blanes

que aplastarlo. Pero nunca dejó de hacerlo. Y ese fue su mérito... (...) ¿Y por qué no escribe un libro sobre esta ciudad y sobre los hombres que vivimos en ella? ¿Y usted, padre, me ayudaría a publicarlo? Se quedó en silencio. Caminamos en silencio un largo trecho. Fue el primero que me habló de un libro. Y la idea quedó germinando desde esa tarde... (1984, IV: 22).

Aparecía, pues, en la parte final del texto, la imagen del 'buen cura', y sus palabras de ánimo se convertían en el origen de la escritura. Gamboa destacaba el nítido alineamiento con la causa de los presos que ordenaba la actitud del capellán, y lo situaba como mediador posible de su testimonio en tanto que perteneciente a un espacio con legitimidad pública. Sin hacer ninguna referencia explícita al trabajo político de la Vicaría de la Solidaridad y de toda una parte de la Iglesia que había reclamado sin tapujos el cese de la violencia, Gamboa leía el gesto del buen capellán en términos de sensibilidad y de piedad cristiana. De ese modo, Gamboa vinculaba sucintamente la escritura de su testimonio con la apertura de espacios públicos para los supervivientes que realizaba la rama descontenta de la Iglesia, aquella que históricamente había pensado la justicia social como uno de los objetivos promulgados por los evangelios.

Ello evitaba situar a su testimonio -explícitamente crítico con las políticas represivas del régimen- como un enfrentamiento directo a las formas de lo decible en Chile, sino que lo articulaba a una de las posibilidades del espacio enunciativo que se había ido tejiendo en el Chile de los ochenta, y en el que los continuados esfuerzos de una parte de la Iglesia católica resultaron de vital importancia. Era, pues, en el espacio abierto por las publicaciones anteriormente señaladas donde el testimonio de Gamboa se inscribía, suponiendo una de las más fuertes apuestas por vincular el trabajo periodístico, la voz traumada de los supervivientes y la ideología política subyacente a las posiciones de la Vicaría de la Solidaridad (verdadera matriz del sentido político de esta serie de testimonios).

Coherentemente con esa vinculación, el texto de Gamboa se cerraba con una potente invocación a la reconciliación nacional. Si la narración se abría con una dedicatoria a su hijo Alberto, a quien señalaba como 'compañero', en el epílogo se nos aclaraba que éste "se graduaba como subteniente en la Escuela Naval Arturo Prat cuando su padre sufría privado de la libertad en el campo de prisioneros de Chacabuco" (1984, IV: 88). Se resaltaba, por tanto, el enfrentamiento de los compromisos de ambos en los meses posteriores al Golpe, y el diferente lugar en que la historia los dejaría con respecto al Régimen Militar. A continuación se nos 
presentaban dos cartas entre hijo y padre en la que ambos se profesaban mutua admiración y leían en su amor incondicional la posibilidad de una reconciliación de los bandos enfrentados en Chile:

Tú, hijo mío, has tenido una formación militar. Yo, tu padre, he tenido la formación que me ha dado el pueblo, mi familia, mis amigos y mi profesión periodística. ¡Yo te encuentro el hijo más maravilloso de la tierra! Tú encuentras en mí un padre del cual, dices, puedes estar orgulloso. ¿Qué cuesta pensar, entonces, que lo que pasa entre nosotros pueda ocurrir entre todos los chilenos? (1984, IV: 95-6).

Así, Gamboa proponía la dinámica de los afectos familiares como el elemento que podía ayudar a superar las divisiones sociales creadas a raíz del Golpe y permitir un cierto entendimiento entre los actores enfrentados en el Chile de los ochenta. El paisaje de lo posible pensado para esa reconciliación era, en este caso, totalmente deudor del imaginario anteriormente señalado en algunas de las intervenciones de la Vicaría y en los libros-reportaje con que se transformó la escena periodística en los primeros ochenta:

Con los años, los rencores y los odios han ido a parar al rincón de los trastos viejos. En todos los organismos se cometen errores. Pero los errores de algunos no pueden comprometer a las instituciones. ¡Que eso quede bien claro! Como tampoco los sufrimientos nuestros son fuentes de rencor o de odio. Por el contrario, pienso que son la semilla de la reconciliación y el reencuentro. ¡Que también eso quede claro!

Todo eso nos quedó grabado mientras vivimos alejados de nuestros hogares, y también les quedó grabado a los hombres de uniforme que estaban tan alejados como nosotros de los suyos. En estas vivencias he destacado muchos gestos anónimos y generosos del soldado o del carabinero hacia este grupo de hombres desesperanzados; como también cientos de abusos y otros atropellos incomprensibles. Era el reencuentro que todos deseábamos, pero que nadie entonces y en ese lugar, se atrevía a confesar. Por eso sueño con que este libro que habla con la franqueza que es, al final de cuentas, la única forma de entenderse, sirva para exterminar los rencores. Que volvamos a encontrarnos con los hombres de las Fuerzas Armadas sin que exista esa alambrada de por medio. (...) Que pensemos nada más que somos chilenos (1984, IV: 95). 
Un viaje por el infierno, de Alberto Gamboa: escritura testimonial e imaginario de la reconciliación / Jaume Peris Blanes

Desde ese punto de vista, que con el tiempo se institucionalizaría y se convertiría en un elemento fundamental de la retórica, el vocabulario y la sintaxis política de la Transición, lo 'opuesto' a la lógica violenta de los militares no era la recomposición de los lazos sociales y de las políticas populares anteriores al Golpe, sino la unidad de los chilenos en un espacio de reconciliación que fuera más allá de las fracturas abiertas por la dictadura. En este caso, era la lógica del afecto paterno-filial la que se proponía como modelo para la convivencia futura, en la que se vieran superados los antagonismos políticos de las décadas pasadas.

\section{Conclusiones}

El testimonio de Gamboa, temprano y valiente, no sólo suponía el entrecruzamiento de los dos imaginarios que habían vertebrado las protestas públicas contra el régimen militar dentro y fuera de Chile (el periodismo y el testimonio de supervivientes), sino que suponía, en un contexto de violencia pública, censura y máxima dificultad editorial, la definitiva inclusión de la narrativa testimonial en el imaginario de la reconciliación.

Gamboa articulaba en su texto algunas de las dificultades enunciativas del testimonio -su débil narratividad, la fragmentación estructural, el juego de distancias entre el sujeto de enunciación y el sujeto del enunciado, la doble temporalidad de la escritura-y algunas de las estrategias retóricas del imaginario de la reconciliación -el paradigma familiar como metáfora de la nación, la sensibilidad y la piedad cristiana como respeto y solidaridad con las víctimas, la ingenuidad ante el carácter político de la violencia represiva-, para crear una intervención pública singular en el contexto de la dictadura militar chilena.

Ello no fue de una importancia menor, pues con el tiempo la retórica de la reconciliación que, en ese contexto turbulento, se había gestado en las intervenciones de la Vicaría y que las publicaciones de los ochenta contribuyeron a expandir y consolidar, acabaría por convertirse, con la vuelta de la democracia, en el lenguaje en el que se expresarían los pactos y compromisos de la Transición democrática. El testimonio de Gamboa se inscribió, tal como hemos demostrado, en ese proceso de consolidación y extensión del imaginario de la reconciliación y en su asociación a los discursos de defensa de los derechos humanos y de denuncia del sistema represivo. Publicaciones posteriores, como Chile: la memoria prohibida (Ahumada et alii, 1989), que este trabajo no ha podido analizar, se encargarían de sellar esa vinculación y de construir

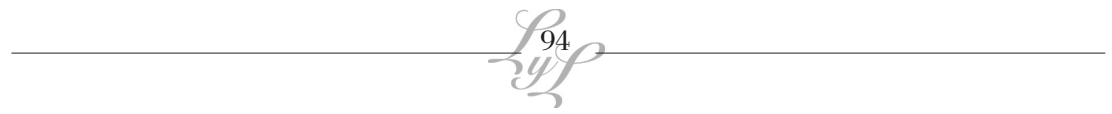


una forma discursiva capaz de abrochar las diferentes intervenciones de denuncia de los ochenta y construir con ellas una sintaxis, un léxico y una estructura narrativa coherente con el imaginario de la reconciliación y con las luchas culturales de la redemocratización. El testimonio de Gamboa, así como los libros-reportaje a los que hacíamos referencia antes, constituyen los antecedentes genealógicos de todo ello.

\section{Bibliografía citada}

Agamben, G. (2000). Lo que queda de Auschwitz. El archivo y el testigo. Homo Sacer III. València: Pre-Textos.

Ahumada, Eugenio; Atria, R.; Egaña, J. L.; Góngora, A.; Quesney, C.; Saball, G.; Villalobos, G. (1989). Chile: La Memoria Prohibida. Santiago de Chile: Pehuén.

Cruz, M. A. (2004). Iglesia, represión y memoria. El caso chileno. Madrid: S. XXI.

Dorfman, A. (1986). "Código político y código literario: el género testimonio en Chile hoy" Testimonio y literatura. (Jara, R.; Vidal, H.) Minessota: Institute for the studies of ideologies and literature: 170-234.

Gamboa, A. (1984). Un viaje por el infierno I. La partida. Santiago de Chile: Libros de Hoy.

(1984b). Un viaje por el infierno II. Chacabuco. Santiago de Chile: Libros de Hoy.

(1984c). Un viaje por el infierno III. Vida de perros. Santiago de Chile: Libros de Hoy.

(1984d). Un viaje por el infierno IV. El regreso. Santiago de Chile: Libros de Hoy.

Langer, L. (1991). Holocaust testimonies. The ruins of memory. New Haven y London: Yale University Press.

García Castro, A. (2002). La mort lente des disparus au Chili. Sous la négociation civils-militaires (1973-2002). París: Maissoneuve \& Larosse.

Klein, N. (2007). La doctrina del shock. El auge del capitalismo del desastre. Barcelona: Paidós.

Moors, X. "Para una arqueología del testimonio: el rol de la iglesia católica en una producción textual (1973-1991)" Revista Iberoamericana LX. 168/169 (1994): 1161-1176. 
Un viaje por el infierno, de Alberto Gamboa: escritura testimonial e imaginario de la reconciliación / Jaume Peris Blanes

Pacheco, M. (1980). Lonquén. Santiago de Chile: Aconcagua.

Politzer, P. (1985). Miedo en Chile. Santiago de Chile: CESOC.

Peris Blanes, J. (2005). La imposible voz. Memoria y representación de los campos de concentración en Chile: la posición del testigo. Santiago de Chile: Cuarto Propio.

(2008). Historia del testimonio chileno. De las estrategias de denuncia a las políticas de memoria. Valencia: Quaderns de Filologia.

Pinto, M. [1984, edición censurada] (1986). Nunca Más Chile. 1973-1984. Santiago de Chile: Terranova Editores.

Scarry, E. (1985). The body in pain. The making and unmaking of the world. New York/Oxford: Oxford University Press.

Sucasas, A. "Anatomía del lager. (Una aproximación al cuerpo concentracionario)" Isegoría 23 (2000): 197-207.

Unnold, Y. (2002). Representing the unrepresentable. Literature of trauma under Pinochet in Chile. New York: Peter Lang.

Verdugo, P.; Orrego, C. [1980, versión censurada] (1983). Detenidosdesaparecidos. Una herida abierta. Santiago de Chile: Aconcagua.

V.V.A.A. (1978). ¿Dónde están? Vol. I. Santiago de Chile: Publicaciones del Arzobispado de Santiago-Vicaría de la Solidaridad. 